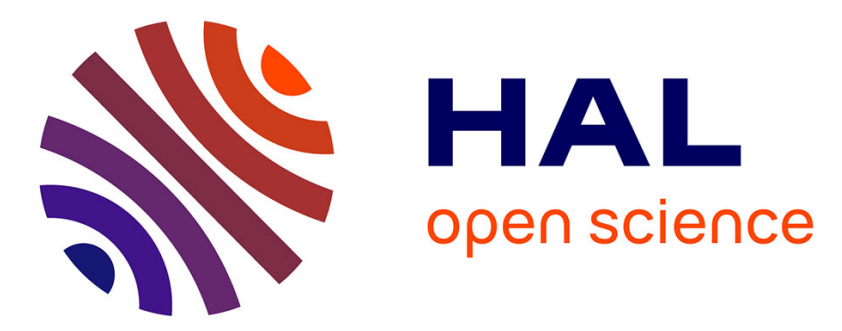

\title{
Simultaneous data reconciliation and parameter estimation. Application to a basic oxygen furnace
}

\author{
Julien Francken, Didier Maquin, José Ragot, Bertrand Bèle
}

\section{To cite this version:}

Julien Francken, Didier Maquin, José Ragot, Bertrand Bèle. Simultaneous data reconciliation and parameter estimation. Application to a basic oxygen furnace. 2nd IFAC International Conference on Intelligent Control Systems and Signal Processing, ICONS 2009, Sep 2009, Istambul, Turkey. pp.CDROM. hal-00395590

\section{HAL Id: hal-00395590 \\ https://hal.science/hal-00395590}

Submitted on 31 Mar 2014

HAL is a multi-disciplinary open access archive for the deposit and dissemination of scientific research documents, whether they are published or not. The documents may come from teaching and research institutions in France or abroad, or from public or private research centers.
L'archive ouverte pluridisciplinaire HAL, est destinée au dépôt et à la diffusion de documents scientifiques de niveau recherche, publiés ou non, émanant des établissements d'enseignement et de recherche français ou étrangers, des laboratoires publics ou privés. 


\section{Simultaneous data reconciliation and parameter estimation. Application to a basic oxygen furnace.}

\author{
Julien Francken, Didier Maquin, José Ragot \\ Centre de Recherche en Automatique de Nancy, \\ UMR 7039 - Nancy-Université, CNRS, \\ 2, Avenue de la forêt de Haye, \\ 54516 Vandouvre-lès-Nancy Cedex, France \\ Email: julien.francken@arcelormittal.com
}

\author{
Bertrand Bèle \\ ArcelorMittal Research Maizières, \\ Voie Romaine \\ 57280 Maizières-lès-Metz, France \\ Email: bertrand.bele@arcelormittal.com
}

\begin{abstract}
In the steel industry, the determination of the control system set-points of batch processes is a common problem. It consists in adjusting the set-points in order to reach the given product specifications thanks to a process model. Small changes in operating conditions may impact final product quality. This is particularly true for the Basic Oxygen Furnace (BOF) where the information collected during a specific batch serves to adjust the set-points of the next batch. For being able to control that type of process, measurements must be made coherent and it may be convenient to use data reconciliation procedure. The proposed paper describes a method allowing simultaneous data reconciliation and model parameter estimation. Parameter estimation results can either be used to update the process model or to detect abnormal parameter variations due, e.g. to fouling, corrosion, degradation of parts of the process.
\end{abstract}

\section{INTRODUCTION}

\section{A. Context}

In the steel industry, the determination of the control system set-points of batch processes is a common problem. It consists in adjusting the set-points in order to reach the given product specifications thanks to a process model. Batch processes are characterized by prescribed processing of raw materials into refined products. The objective is to produce products of the desired quality. However, small changes in operating conditions may impact final product quality. Moreover, batch processes with the same trajectory generally exhibit some degree of batch-to-batch variation. This is particularly true for the Basic Oxygen Furnace (BOF) where the information collected during a specific batch serves to adjust the set-points of the next batch. Usually measurements collected from an industrial process contain random errors, systematic biases or gross errors due to the quality of the instrumentation chain and its environment. Based on first principles (mass and energy balances, chemical equilibrium relationships, etc), modelfiltering approach, commonly called data reconciliation may be used to provide coherent data. However, the main hypothesis underlying these methods is that the model describing the system is perfectly known which is not the case in real world. The proposed paper describes a method allowing simultaneous data reconciliation and model parameter estimation.

\section{B. The Basic Oxygen Furnace}

Basic oxygen furnace is a method of primary steelmaking in which carbon-rich molten iron is made into refined steel. The vast majority of steel manufactured in the world is produced using the basic oxygen furnace. Modern furnaces will take a charge of iron of up to 350 tons and convert it into steel in less than 40 minutes.

By blowing oxygen through molten pig iron, the carbon content of the alloy is lowered and changes the material into low-carbon steel.

The basic oxygen furnace actions are scheduled as follows: molten iron from a blast furnace is poured into a large refractory-lined container. Then the container is filled with the required ingredients which quantities are computed thanks to a setup charge balance model. As the required thermal energy is produced during the process, it is relevant to maintain a proper charge balance.

A water-cooled lance is so lowered down into the vessel to blow pure oxygen onto the steel and iron, igniting the carbon dissolved in the steel and burning it to form carbon oxide, causing the temperature to rise to about $1700^{\circ} \mathrm{C}$. This melts the scrap, lowers the carbon content of the molten iron and helps remove unwanted chemical elements. Other fluid components are added to form slag which absorbs impurities of the steelmaking process and emulsifies to make easier the refining process. At the end of the blowing cycle, the temperature is measured and samples are taken to give a chemical analysis.

The main functions of the BOF are to decarburize and remove phosphorus from the hot metal, and to optimize the steel temperature so that any further treatments prior to casting can be performed with minimal reheating or cooling of the steel. These actions are essentially performed by blowing oxygen with high pressure on hot metal and adding scraps and iron ore. The exothermic oxidation reactions that occur during BOF process generate a lot of heat energy, more than is necessary to attain the target steel temperature. This extra heat is used to melt scrap and/or iron ore additions.

For the BOF, the setup problem consists to determine 
the quantity of iron ore to add and the oxygen volume to blow to reaching the target of Carbon rate in steel and the temperature defined for each heat (batch) by the given product specification.

This task is done by a static charge computation based on a model formed by comprehensive heat and mass balances. These balances between input and output products describe the system. For example, a simplified iron mass balance equation could be as follows:

$$
[\% \mathrm{Fe}]_{S c p} Q_{S c p}+[\% \mathrm{Fe}]_{H M} Q_{H M}-[\% \mathrm{Fe}]_{S t l} Q_{S t l}=0
$$

with

- $[\% \mathrm{Fe}]_{S c p}$ : the iron mass percentage in scraps

- $[\% \mathrm{Fe}]_{H M}$ : the iron mass percentage in hot metal

- $[\% \mathrm{Fe}]_{S t l}$ : the iron mass percentage in steel

- $Q_{S c p}$ : the scrap quantity to convert

- $Q_{H M}$ : the hot metal quantity to convert

- $Q_{S t l}$ : the converted steel quantity

For each heat, the system state variables have a different operating point.

\section{Main objectives and proposed method}

Data reconciliation and parameter estimation are fundamental components to real time optimization of industrial processes. Great efforts have been made to develop models for various processes. However, as mentioned by Rolandi and Romagnoli [6], physical and chemical process phenomena are complex and difficult to model conceptually and mathematically. Indeed, thermodynamic and transport properties and reaction rates are difficult to characterize experimentally and are subject to parametric uncertainties. Moreover, even when measurements are sufficiently numerous, raw processinstrumentation data are also corrupted by different errors. Indeed, data sets taken at a certain operation point are not model-consistent. Online optimization provides a mean to run a process near its optimum operating condition by providing real-time computed optimal set-points to the control system [3]. However, the implemented algorithms need to be fed by coherent data. The more classical way to deal with that problem consists to reconcile process data based on the minimization of measurement errors subject to satisfying the model constraints and next estimate the model parameters from these reconciled values. An alternative to this twostep approach consists to develop simultaneous strategies for data reconciliation and parameter estimation [5], [7], [4]. The proposed paper is dedicated to the presentation of such method.

On a mathematical point of view, data reconciliation is generally based on the assumption that the measurement errors have Gaussian probability density function (pdf) with zero mean. As some model parameters are uncertain, they are also modeled as random Gaussian variables.

Simultaneous state variable and parameter estimates are obtained using a maximum likelihood estimation approach applied on a sliding time window of observation data. To make less sensitive the parameter estimation to measurement uncertainties, the parameters are considered as constant value on a given time window.

Parameter estimation results can either be used to update process model or to detect abnormal variation due, e.g. to fouling, corrosion, degradation of parts of the process.

\section{Simultaneous DATA RECONCILIATION AND PARAMETER ESTIMATION}

The proposed paper describes a method allowing simultaneous data reconciliation and model parameter estimation in order to provide model-consistent data. In real process, model parameters are subjected to smooth evolution/degradation, that's why simultaneous state variable and parameter estimates will be extracted from a sliding time window of observation data. For each observation data window, parameters are assumed to be constant whereas state variables evolve around different operating points. This computation artifice will make less sensitive the parameter estimates to measurement uncertainties.

The method of simultaneous data reconciliation and parameter estimation on a given window of length $N$ is firstly presented and the corresponding algorithm is outlined. Next, the practical implementation using a sliding window is described

\section{A. The process model}

Consider the system described by:

- a set of nonlinear equations in relation to a vector of state variables $x_{i}^{*}$ and a parameter vector with true value $a^{*}$

$$
\begin{gathered}
F\left(x_{i}^{*}, a^{*}\right)=0, \quad i \in[1, N] \\
x_{i}^{*} \in \mathbb{R}^{v}, \quad a^{*} \in \mathbb{R}^{p}, \quad F: \mathbb{R}^{v \times p} \rightarrow \mathbb{R}^{n}
\end{gathered}
$$

- observation equations

$$
x_{i}=x_{i}^{*}+\varepsilon_{x i}, \quad i \in[1, N]
$$

- a priori knowledge of the parameters

$$
a=a^{*}+\varepsilon_{a}
$$

The $x_{i}$ measurements of the true values $x_{i}^{*}$ are available for the $N$ realizations. For the parameter, the a priori knowledge is formulated as a kind of "observation equation". The parameter is defined on the basis of a nominal value and a "pseudonoise". This form allows to express in the same manner, the state variable and parameters probability density functions, given as follows:

$$
\begin{aligned}
p_{x i} & =\frac{1}{(2 \pi)^{v / 2}|V|^{1 / 2}} \exp \left(-\frac{1}{2}\left(x_{i}^{*}-x_{i}\right)^{T} V^{-1}\left(x_{i}^{*}-x_{i}\right)\right) \\
p_{a} & =\frac{1}{(2 \pi)^{p / 2}|W|^{1 / 2}} \exp \left(-\frac{1}{2}\left(a^{*}-a\right)^{T} W^{-1}\left(a^{*}-a\right)\right)
\end{aligned}
$$

with $V$ and $W$ the variance-covariance matrices of the state variables and parameters respectively. The measurement and 
parameter error distributions are then assumed independent random variables.

\section{B. State and parameter estimation method}

The problem issue concerns the estimation of the true value of the state variables and the model parameters based on the knowledge of the measurements (3) on the given horizon $[1, N]$, the a priori knowledge (4) of the parameter and the process model (2). The estimation can be performed by the maximum likelihood principle. Taking into account that measurement errors are independent, the likelihood function $\mathcal{V}$ is the product of the probability density functions:

$$
\mathcal{V}=\prod_{i=1}^{N} p_{x i} p_{a}
$$

Estimations $\hat{x}_{i}, \hat{a}$ of the true values maximize the likelihood function $\mathcal{V}$ in relation to $x_{i}^{*}$ and $a^{*}$ with respect to the constraint (2) satisfaction.

Let $\Phi$ be the Lagrange function relative to the log-likelihood function and the constraint that have to be satisfied:

$$
\Phi=\ln \mathcal{V}+\sum_{i=1}^{N} \lambda_{i}^{T} F\left(x_{i}^{*}, a^{*}\right)
$$

with $\lambda_{i} \in \mathbb{R}^{n}$.

Genrally, this nonlinear optimisation problem must be solved using an iterative algorithm solution. In this case, instead of a global system linearization arised from Lagrange regularity conditions, it is advisable to previously linearize the constraint equations.

For all the realizations $i \in[1, N]$, at the iteration $j$, we assume that a solution pair $\left\{\hat{x}_{i, j}, \hat{a}_{j}\right\}$ is available. From this solution pair, estimates can be improved based on a first order Taylor series expansion in the neighborhood of this solution pair. Assuming the convergence of the estimation process, the successive solution pairs constitute a convergent series toward the problem solution.

At the iteration $j+1$, for a solution pair $\left\{\hat{x}_{i, j+1}, \hat{a}_{j+1}\right\}$, in the neighborhood of $\left\{\hat{x}_{i, j}, \hat{a}_{j}\right\}$, the constraint first order Taylor series is:

$$
\begin{aligned}
F\left(\hat{x}_{i, j+1}, \hat{a}_{j+1}\right)= & F\left(\hat{x}_{i, j}, \hat{a}_{j}\right)+G_{x}\left(\hat{x}_{i, j}, \hat{a}_{j}\right)\left(\hat{x}_{i, j+1}-\hat{x}_{i, j}\right) \\
& +G_{a}\left(\hat{x}_{i, j}, \hat{a}_{j}\right)\left(\hat{a}_{j+1}-\hat{a}_{j}\right)
\end{aligned}
$$

with the constraint gradients as follows:

$$
\begin{aligned}
& G_{x}\left(\hat{x}_{i, j}, \hat{a}_{j}\right)=\left.\frac{\partial F\left(x_{i}^{*}, a^{*}\right)}{\partial x_{i}^{* T}}\right|_{x_{i}^{*}=\hat{x}_{i, j}, a^{*}=\hat{a}_{j}} \\
& G_{a}\left(\hat{x}_{i, j}, \hat{a}_{j}\right)=\left.\frac{\partial F\left(x_{i}^{*}, a^{*}\right)}{\partial a^{* T}}\right|_{x_{i}^{*}=\hat{x}_{i, j}, a^{*}=\hat{a}_{j}}
\end{aligned}
$$

For more readability, these last expressions will be denoted $F_{i}, G_{i x}, G_{i a}$ omitting the dependancy with regard to the quantities at iteration index $j$. At iteration $j+1$, estimation problem reduces to the search of the $\Phi_{j+1}$ Lagrange function extremum in relation to $x_{i, j+1}^{*}, a_{j+1}^{*}$, and $\lambda_{i}$ :

$$
\begin{aligned}
\Phi_{j+1} & =\Phi_{0}-\frac{1}{2}\left(a_{j+1}^{*}-a\right)^{T} W^{-1}\left(a_{j+1}^{*}-a\right) \\
& -\frac{1}{2} \sum_{i=1}^{N}\left(x_{i, j+1}^{*}-x_{i}\right)^{T} V^{-1}\left(x_{i, j+1}^{*}-x_{i}\right) \\
& +\sum_{i=1}^{N} \lambda_{i}^{T}\left(F_{i}+G_{i x}\left(x_{i, j+1}^{*}-\hat{x}_{i, j}\right)+G_{i a}\left(a_{j+1}^{*}-\hat{a}_{j}\right)\right)
\end{aligned}
$$

where $\Phi_{0}$ is the constant component of Lagrange function.

This Lagrange function presents an extremum for $x_{i, j+1}^{*}=$ $\hat{x}_{i, j+1}$ and $a_{j+1}^{*}=\hat{a}_{j+1}$ if:

$$
\begin{aligned}
\frac{\partial \Phi_{j+1}}{\partial x_{i, j+1}^{*}} & =0 \\
\frac{\partial \Phi_{j+1}}{\partial a_{j+1}^{*}} & =0 \\
\frac{\partial \Phi_{j+1}}{\partial \lambda_{i}} & =0
\end{aligned}
$$

Equation (12) can be transformed into the following system:

$$
\begin{array}{r}
-V^{-1}\left(\hat{x}_{i, j+1}-x_{i}\right)+G_{i x}^{T} \lambda_{i}=0 \\
-W^{-1}\left(\hat{a}_{j+1}-a\right)+\sum_{i=1}^{N} G_{i a}^{T} \lambda_{i}=0 \\
F_{i}+G_{i x}\left(\hat{x}_{i, j+1}-\hat{x}_{i, j}\right)+G_{i a}\left(\hat{a}_{j+1}-\hat{a}_{j}\right)=0
\end{array}
$$

From (13), (14) and (15), one can deduce:

$$
\begin{aligned}
\hat{x}_{i, j+1} & =x_{i}+V G_{i x}^{T} \lambda_{i} \\
\hat{a}_{j+1} & =a+W \sum_{i=1}^{N} G_{i a}^{T} \lambda_{i} \\
G_{i x} \hat{x}_{i, j+1} & =G_{i x} \hat{x}_{i, j}-F_{i}-G_{i a}\left(\hat{a}_{j+1}-\hat{a}_{j}\right)
\end{aligned}
$$

The Lagrange parameters can be deduced from (16) and (18):

$\lambda_{i}=\left(G_{i x} V G_{i x}^{T}\right)^{-1}\left(G_{i x}\left(\hat{x}_{i, j}-x_{i}\right)-F_{i}-G_{i a}\left(\hat{a}_{j+1}-\hat{a}_{j}\right)\right)$

Substituting (19) in (17) gives:

$\hat{a}_{j+1}=a+W \sum_{i=1}^{N} S_{i}\left(G_{i x}\left(\hat{x}_{i, j}-x_{i}\right)-F_{i}-G_{i a}\left(\hat{a}_{j+1}-\hat{a}_{j}\right)\right)$

with $S_{i}$ defined by:

$$
S_{i}=G_{i a}^{T}\left(G_{i x} V G_{i x}^{T}\right)^{-1}
$$

Let us define $R$ as follows:

$$
R=I+W \sum_{i=1}^{N} G_{i a}^{T}\left(G_{i x} V G_{i x}^{T}\right)^{-1} G_{i a}
$$


Assuming that the matrix $R$ is regular,

$$
\begin{aligned}
\hat{a}_{j+1}= & R^{-1} W \sum_{i=1}^{N} S_{i}\left(G_{i x}\left(\hat{x}_{i, j}-x_{i}\right)-F_{i}+G_{i a} \hat{a}_{j}\right) \\
& +R^{-1} a
\end{aligned}
$$

Finally the expression of the state estimation is obtained from (16) and (19):

$$
\begin{array}{r}
\hat{x}_{i, j+1}=x_{i}+V G_{i x}^{T}\left(G_{i x} V G_{i x}^{T}\right)^{-1}\left(G_{i x}\left(\hat{x}_{i, j}-x_{i}\right)\right. \\
\left.-F_{i}-G_{i a}\left(\hat{a}_{j+1}-\hat{a}_{j}\right)\right)
\end{array}
$$

The estimations $\hat{x}_{i}$ and $\hat{a}$ reliant to $x_{i}$ and $a$ are obtained using successive iterations provided algorithm convergence (the analysis of this latter can be based on the norms of the gradient matrices $G_{i x}$ and $G_{i a}$ ).

\section{Algorithm}

For an observation data window of length $N$, an algorithm based on the method developped in the previous section could be formulated in the following way:

- Initialization

$$
j=0, \forall i \in[1 ; N], \hat{x}_{i, j}=x_{i} \text { and } \hat{a}_{j}=a
$$

- Repeat

Compute $F_{i}, G_{i x}$ and $G_{i a}$ using (9), (10a) and (10b).

Compute $\hat{a}_{j+1}$ using (23)

Compute $\hat{x}_{i, j+1}$ using (24)

$j=j+1$

until norms of $G_{i x}$ and $G_{i a}$ are below a given threshold.

- Update the parameter and variable estimates

$\hat{a}=\hat{a}_{j+1}$

$\hat{x}_{i}=\hat{x}_{i, j+1}, \forall i \in[1 ; N]$

This algorithm gives coherent parameter and state estimates for a given observation data window of length $N$. Let us now examine the practical implementation of this algorithm.

\section{Practical implementation - Estimation on a sliding window}

In order to be able to monitor the time evolution of the model parameters, the proposed algorithm is implemented using a sliding window. Let us consider a first observation window of length $N$. Let us denote $a^{(N)}$ the a priori nominal value of the parameter vector (given by the user) for this observation window. From the knowledge of $X_{1}, \ldots, X_{N}$ and $a^{(N)}$, the proposed algorithm provides the estimates $\hat{X}_{1}, \ldots, \hat{X}_{N}$ and $\hat{a}^{(N)}$. The observation window is then slid from one observation. Based on the knowledge of the measurements $X_{2}, \ldots, X_{N+1}$ and the previously estimated value of parameter vector $\hat{a}^{(N)}$ which serves as the nominal value for this new observation data window, the algorithm is used to provide the estimates $\hat{X}_{N+1}$ and $\hat{a}^{(N+1)}$. This process, which is depicted on figure 1 is re-iterated based on the availability of new sets of measurements along the time.

As previously said, the determination of the control system set-points of batch processes in the steel industry is difficult to solve. Indeed, small changes in the operating conditions

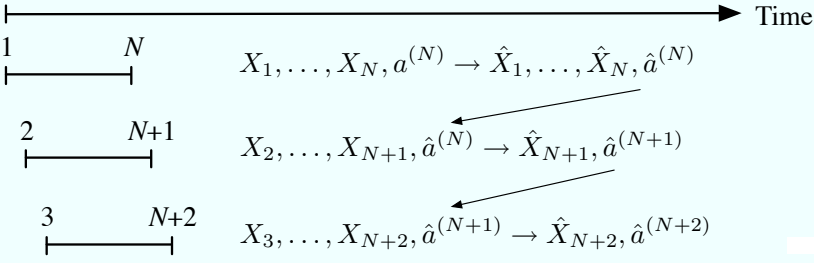

Fig. 1. Principle of estimation on a sliding window

may impact final product quality and some degree of batch-tobatch variation exists. Therefore, model adaptation algorithm are frequently implemented for that kind of processes. Clearly, the performances of the existing model adaptation algorithm will be enhanced when fed by the coherent estimated values provided by the proposed method.

\section{EXAMPLE: SIMULTANEOUS STATE AND PARAMETER ESTIMATIONS OF A SIMPLIFIED BOF PROCESS}

The proposed simultaneous state and parameter estimation method has been applied to a simplified nonlinear process model of a basic oxygen furnace (BOF).

\section{A. The process model}

Due to the random nature of chemical reaction in the non homogeneous slag or to the wear-out refractory-lined container or other many causes, the process cannot accurately be described with equilibrium balances. Therefore some of the model parameters need to be estimated without forget to use model consistent datas.

As described in section I-A, the model is a set of mass and heat balances. Let the nonlinear equation system be a simplified model of basic oxygen furnace described as next:

$$
\begin{array}{r}
0.5 x_{1}^{*}+\left(-3+x_{2}^{*}\right) x_{3}^{*}+\left(a_{1}^{*}-x_{4}^{*}\right) x_{5}^{*}=0 \\
3 x_{1}^{*}+\left(0.25 x_{2}^{*} x_{4}^{*}-x_{5}^{*}\right) x_{3}^{*}+9=0 \\
x_{1}^{*}-0.5 x_{2}^{*} x_{3}^{*}+x_{4}^{*}+a_{2}^{*} x_{5}^{*}-1=0
\end{array}
$$

The first equation represents the iron mass balance where $x_{1}, x_{3}, x_{5}$ are material quantities, $x_{2}$ and $x_{4}$ iron mass percentage and $a_{1}$ a parameter which evolves along the time. The second equation describes a heat balance and the third another chemical element mass balance.

We have at our disposal state variable measurements $x_{i}$ for 1000 realizations. Their accuracies (constant for the whole realizations) are given in the table I. For that example, it is desired to monitor the values of the two parameters $a_{1}^{*}$ and $a_{2}^{*}$, the others being assumed constant. A priori knowledge on $a_{1}$ and $a_{2}$ parameters are gathered in table II.

\section{B. Results}

To validate the proposed method, a data base composed of measurement data and true values of evolving parameters have been created for 1000 realizations. The simultaneous 


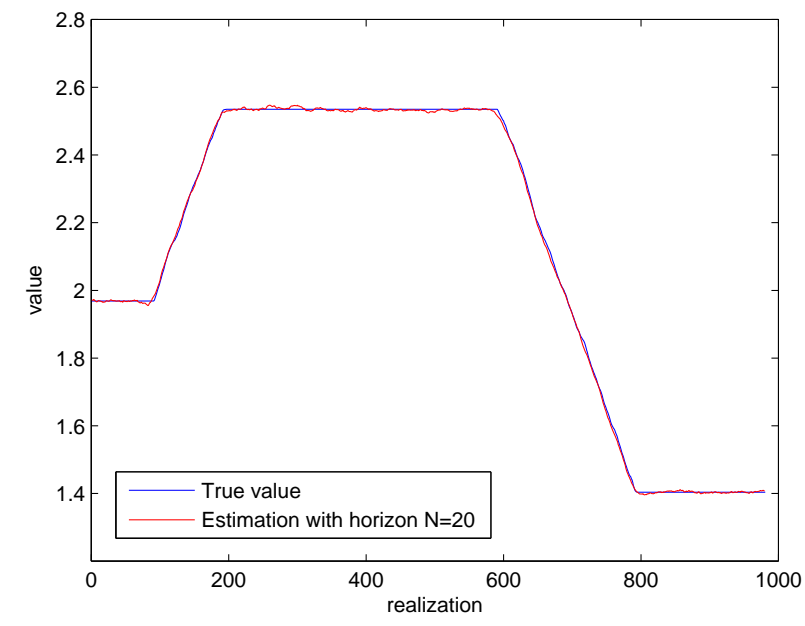

Fig. 2. $a_{1}$ parameter estimation

state and parameter estimation algorithm described above was performed based on the measurement data from the whole realizations, the knowledge of the model, and only parameter nominal values given in table II. Because data proceed from created data base, we can easily observe the performance of the parameter estimation by matching parameter estimates with the true values. Results are given in figure 2 and 3 when the observation data window length is fixed to $N=20$, parameters estimates are near to the true value. Figure 4 shows a zoom (realizations 50 to 500) on the estimation of parameter $a_{2}$ when using different lengths for the sliding time window. The filtering capacity of the proposed estimator is pointed out. The more the window length is bigger, the more the parameter estimation filter the noise measurement, but, in the same time, the more the estimation is delayed.

Figures 5, 6, 7, 8, 9 show the measurements of the state variables and their estimations. As the measurements are unbiased, the estimations are closed to the measurements.

\section{CONCLUSION}

In this paper, a general methodology for simultaneous data reconciliation and parameter estimation for nonlinear model has been proposed. Data reconciliation based on balances was performed to obtain model-consistent measurement data and simultaneously parameter estimates. The use of a sliding window on which parameters are considered as constant values allows the desensitization of their estimations with regard measurement uncertainties. It is interesting to note that the

\begin{tabular}{lccccc}
\hline Variable & $x_{1}$ & $x_{2}$ & $x_{3}$ & $x_{4}$ & $x_{5}$ \\
\hline Standard deviation & 0.033 & 0.16 & 0.2 & 0.11 & 0.23 \\
& TABLE I \\
VARIABLE MEASUREMENT ACCURACIES &
\end{tabular}

VARIABLE MEASUREMENT ACCURACIES

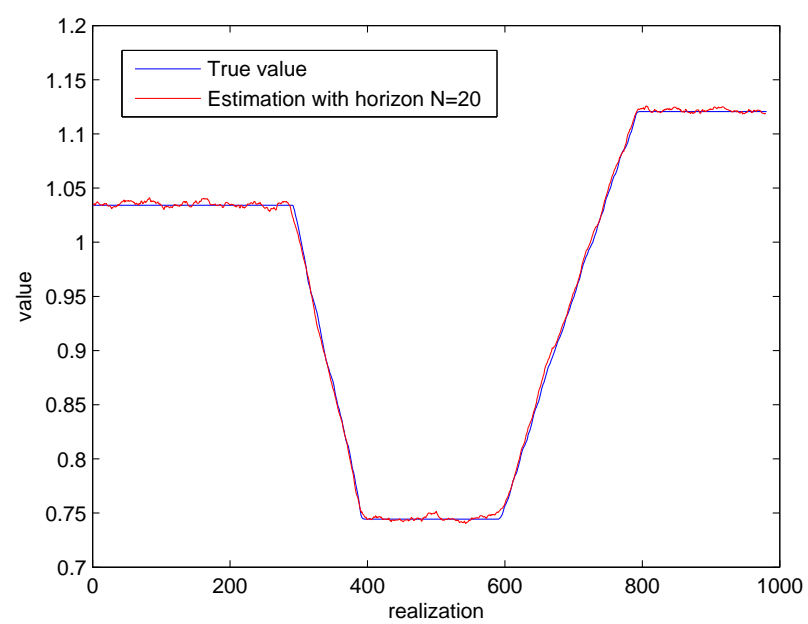

Fig. 3. $a_{2}$ parameter estimation

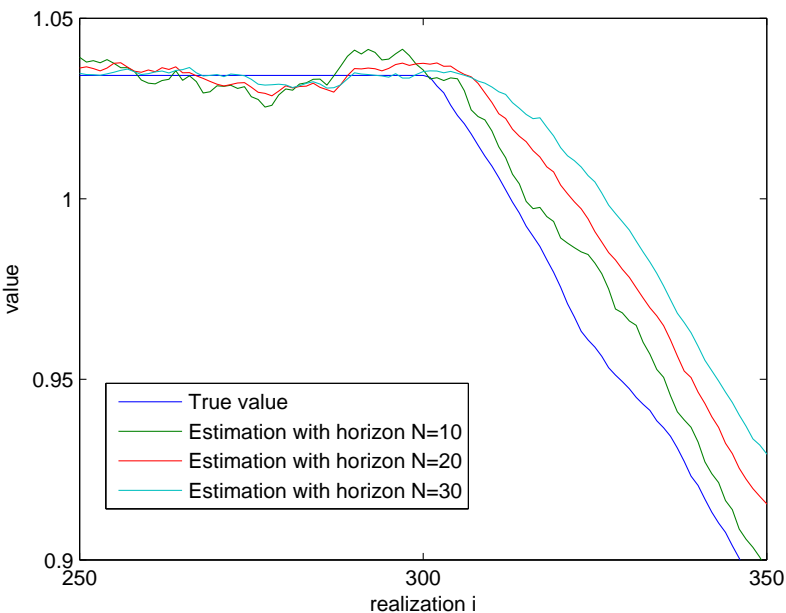

Fig. 4. Width window impact on parameter estimation
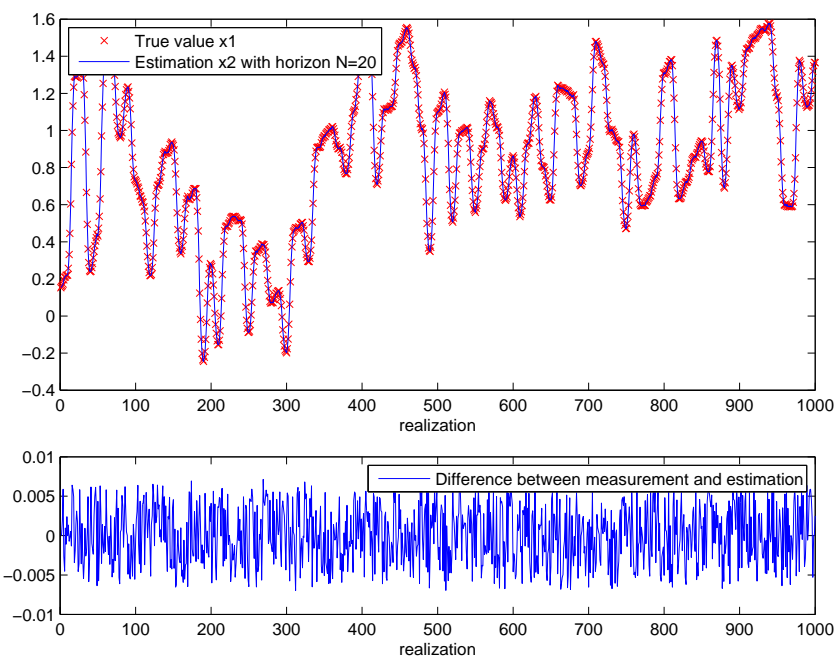

Fig. 5. $x_{1}$ state estimation and measurement 

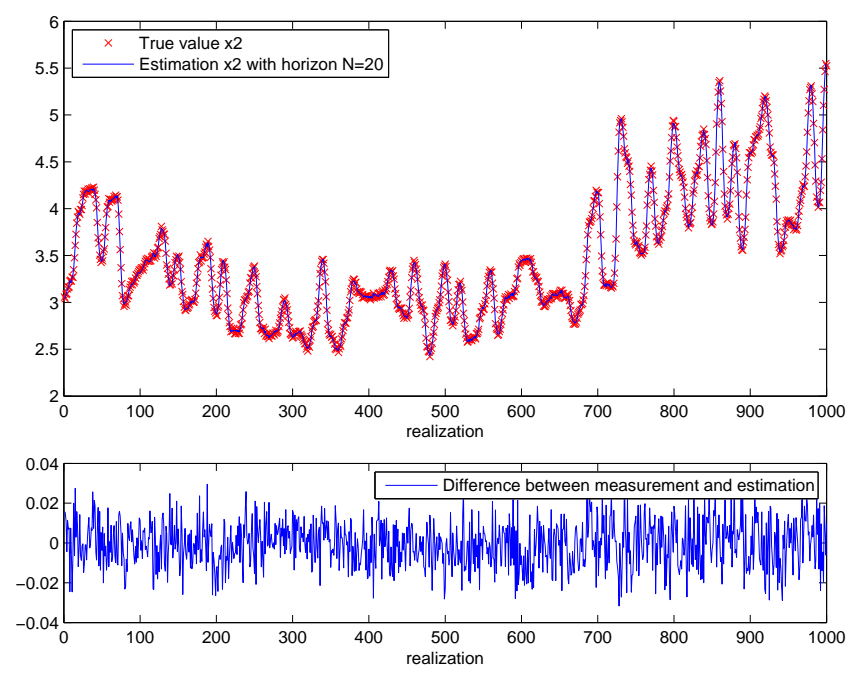

Fig. 6. $x_{2}$ state estimation and measurement
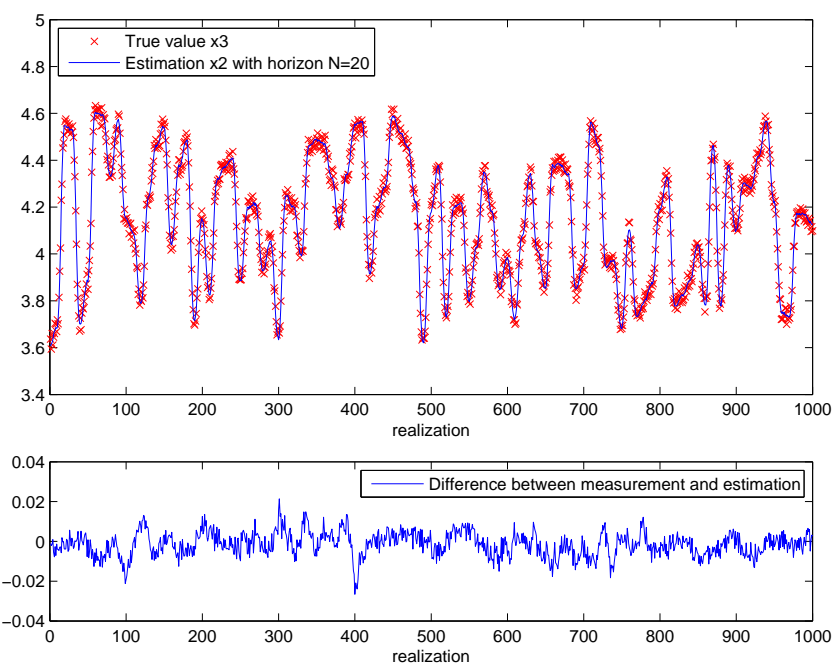

Fig. 7. $x_{3}$ state estimation and measurement
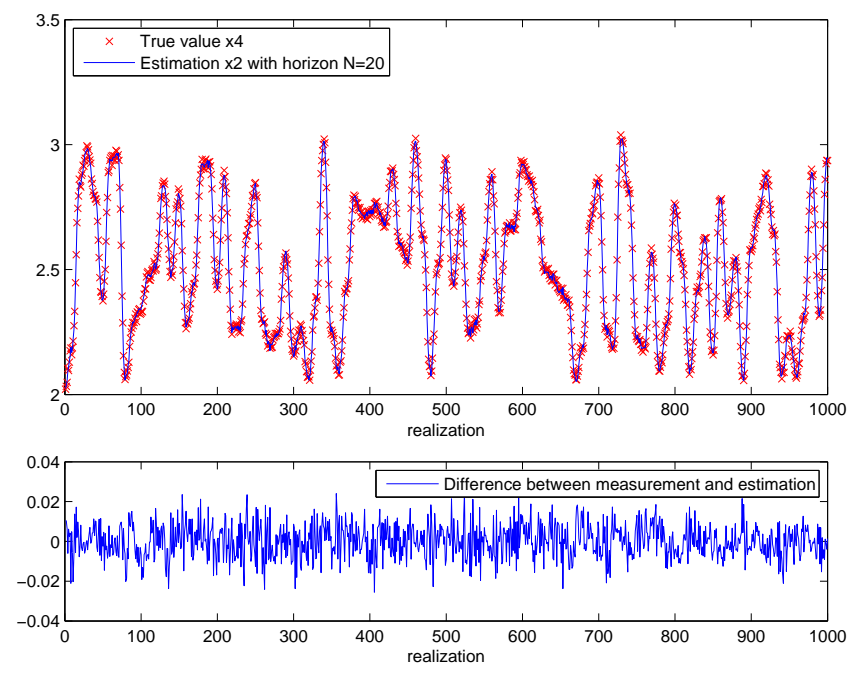

Fig. 8. $x_{4}$ state estimation and measurement

\begin{tabular}{lcc}
\hline Parameter & $a_{1}$ & $a_{2}$ \\
\hline Nominal value & 2 & 1 \\
Standard deviation & 0.1 & 0.05
\end{tabular}

TABLE II

"A PRIORI" PARAMETER KNOWLEDGE
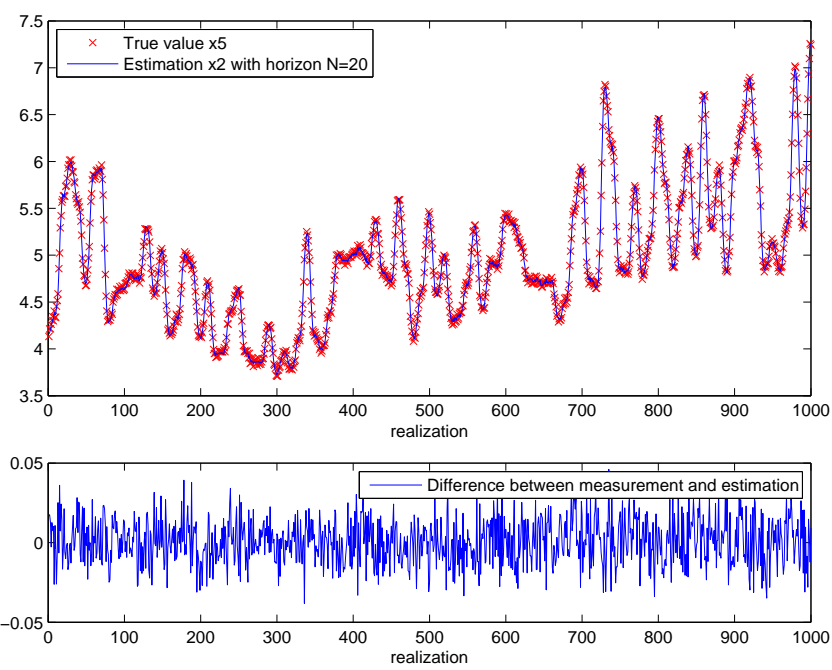

Fig. 9. $x_{5}$ state estimation and measurement

knowledge of parameter distribution errors is not a limiting factor, indeed it would be easy to modify the algorithm in order to run without any parameter knowledge. The next step would be to include some robustness properties with regard gross measurement errors using a so-called contaminated distribution in the maximum likelihood estimation [2], [1]. Finally the proposed method must be evaluated on real process model with real data in terms of process model adjustment along the successive heat to observe the impact of the setpoints adjustment on the successive batch processes.

\section{REFERENCES}

[1] M. Alhaj-Dibo, D. Maquin, J. Ragot, Data reconciliation: a robust approach using contaminated distribution. Control Engineering Practice, 16(2):159-170, 2008.

[2] N. Arora, L.T. Biegler, Redescending estimators for data reconciliation and parameter estimation. Chemical Engineering and Processing, 25:1585-1599, 2001

[3] R. Faber, H. Arellano-Garcia, P. Li, G. Wozny, An optimization framework for parameter estimation of largscale systems. Chemical Engineering and Processing, 46:1085-1095, 2007.

[4] Y.Y. Joe, D. Wang, J. Romagnoli, A robust strategy for joint data reconciliation and parameter estimation. European Symposium on Computer Aided Process Engineering, ESCAPE-14, Lisbon, Portugal, May 16-19, 2004.

[5] V. Rod, V. Hancil, Iterative estimation of model parameters when measurements of all variables are subject to error. Computers \& Chemical Engineering, 4(2):33-38, 1980.

[6] P.A. Rolandi, J.A. Romagnoli, Simultaneous dynamic validation/identification of mechanistic process models and reconciliation of industrial process data. Computer Aided Chemical Engineering, 21(A):267-272, 2006.

[7] B. Tjoa, L.T. Biegler, Simultaneous strategies for data reconciliation and gross error detection of nonlinear systems. Computers \& chemical engineering, 15(10):679-690, 1991. 